\title{
How face masks influence the sinonasal quality of life during the COVID-19 pandemic
}

\author{
Adi Primov-Fever ${ }^{1,2} \cdot$ Ofer Amir ${ }^{2,3} \cdot$ Ilan Roziner $^{2,3} \cdot$ Ramit Maoz-Segal ${ }^{2,4} \cdot$ Eran Emanuel Alon $^{1,2}$. \\ Arkadi Yakirevitch ${ }^{1,2}$ (1)
}

Received: 19 February 2021 / Accepted: 11 March 2021 / Published online: 27 March 2021

(c) The Author(s), under exclusive licence to Springer-Verlag GmbH Germany, part of Springer Nature 2021

\begin{abstract}
Purpose To study different mask types' impact on a sinonasal quality of life.

Methods For this observational cross-sectional study, a web-based survey was distributed via social media forums. We used the validated Hebrew version of the Sinonasal Outcome Test-22 followed by a questionnaire developed specifically for the present study, focusing on the time of the COVID-19 pandemic (Mask Sinonasal Outcome Test), and questions regarding general health issues. The participants' mask-wearing routine was also studied.

Results Seventy percent of 351 participants had experienced a change in their breathing during the time of the pandemic. The median total Sinonasal Outcome Test-22 score was 13 , and $10 \%$ of the participants reported a significantly impaired quality of life. According to multivariate analyses, the only subject-related variables significantly associated with the reduced sinonasal quality of life were female gender, younger age, a background of chronic rhinitis and sinusitis, and the mask-wearing average daily duration. The mask sinonasal outcome test convergent validity was confirmed.

Conclusion The majority of our survey's responders, predominantly female and younger participants, reported reduced sinonasal quality of life in the COVID-19 pandemic period. It can be attributed to mask-wearing, especially for a prolonged time, irrespective of the existing mask type. These findings should encourage medical companies to produce more "airwayminded" personal protection equipment.
\end{abstract}

Keywords COVID-19 $\cdot$ Masks $\cdot$ Quality of life $\cdot$ Questionnaires $\cdot$ Sino-nasal outcome test

\section{Introduction}

Due to the COVID-19 pandemic outbreak, large populations worldwide are obliged to wear face masks. This obligation is enforced outdoors, in public places, and in workplaces, depending on local policies. Consequently, many people are required to wear a mask continuously for long hours. This unusual condition has been accompanied by a shortage of personal protective equipment in many places. This shortage evoked the use of improvised masks and non-authorized production, with varied and unknown quality [1]. Using such equipment may harm the sinonasal quality of life $(\mathrm{QoL})$,

1 Department of Otolaryngology and Head and Neck Surgery, The Chaim Sheba Medical Center, 52621 Tel-Hashomer, Israel

2 The Sackler School of Medicine, Tel Aviv University, Tel Aviv, Israel

3 Department of Communication Disorders, The Stanley Steyer School of Health Professions, Tel-Aviv University, Tel-Aviv, Israel

4 Clinical Immunology, Angioedema and Allergy Unit, The Zabludowicz Center for Autoimmune Diseases, Sheba Medical Center, Tel Hashomer, Israel

Eran Emanuel Alon

Eran.Alon@sheba.health.gov.il 
among other reasons, through inhalation of fabric dust entrapped in the masks and subsequent mucosal reaction [2]. This mechanism was related to the increased prevalence of rhinitis and nasal polyposis among textile workers [3, 4]. In contrast, Dror et al. have shown that both standard surgical masks and N95 respirators may improve nasal symptoms of intermittent allergic rhinitis [5]. To the best of our knowledge, the latter study is the only one on the effect of facial mask-wearing on the sinonasal QoL.

We used the validated Hebrew version of the Sinonasal Outcome Test-22 (SNOT-22) to study different mask types' impact. This questionnaire addresses various aspects directly related to specific diseases of the nose and sinuses, as well as general health [6]. Previous studies have confirmed that the SNOT-22 is valid and reliable for this purpose. It is the preferred instrument for evaluating patients with sinonasal diseases, compared to other QoL questionnaires [7, 8]. In a subsequent study, the SNOT-22 questionnaire was further broken down into three sinus-specific symptom domains (rhinologic, extranasal rhinologic, and ear/facial symptoms) and two general health-related QoL domains (psychological and sleep dysfunction) [9]. In line with this approach, the present study evaluates how facial masks affect sino-nasal QoL in people of different ages, gender, and medical backgrounds, particularly pulmonary and sino-nasal problems.

\section{Methods}

This observational cross-sectional study was approved by the Tel-Aviv University Ethics Committee (\#1-0002166). A web-based survey was distributed via social media forums (i.e., Facebook, WhatsApp), and potential participants were approached using a snowball sampling method. The survey was anonymous, and responders were allowed to terminate their participation at any time. All participants provided informed consent before initiating the survey and only then completed the survey. As the COVID-19 pandemic created a unique and highly dynamic situation, it was deemed necessary to limit the time allocated for data collection to a short period. Consequently, all questionnaires were completed between September 29 to October 7, 2020. During this period, local regulations required to wear a mask on the street, at work, and while shopping. The only exceptions were outdoor sports activities and staying at home with household members only.

The survey questionnaire (Appendix 1) opened with an initial question, inquiring whether during the COVID-19 pandemic the participant had experienced a change in nasal breathing (with possible responses of "Yes", "Maybe", and "No"). This question was followed by two outcome scales: (a) the SNOT-22 (items 2-23, Appendix 1), and (b) a questionnaire developed specifically for the present study, with a specific focus on the time of the COVID-19 pandemicmask sinonasal outcome test (MaskSNOT) (items 24-30, Appendix 1). Its seven questions focused on sensations that might occur when using a face mask for extended times, such as nasal itching and a need for nose-picking. Responses to these items were given on a 6-point scale, similar to the SNOT-22 and ranging from $0=$ "Not a problem" to $5=$ "A very severe problem". Summative scores were computed for the whole SNOT-22 questionnaire, with Chronbach's internal consistency coefficient of $\alpha=0.93$. Cronbach's alphas for the five sub-domains of the questionnaire were as follows: rhinologic symptoms $(\alpha=0.85)$, extranasal rhinologic symptoms $(\alpha=0.79)$, ear/facial symptoms $(\alpha=0.78)$, psychological dysfunction $(\alpha=0.90)$, and sleep dysfunction $(\alpha=0.91)$. Finally, the overall score of the MaskSNOT questionnaire was calculated similarly, as a sum of its seven items (Cronbach's $\alpha=0.85$ ).

The predictor measures included a question regarding general health issues: whether the participants receive continuous medical treatment due to the chronic airway problem. The participants were also asked about the preferred mask type, a number of times they changed their facial masks during the day, the average daily number of hours they were wearing a mask, and the frequency of wearing a mask in six daily life situations ( 0 -not at all; 1 -rarely; 2-sometimes; 3-often; 4-always).

Statistical analyses were performed with SPSS v.25 software. Descriptive statistics of research variables were calculated as percentages or means and standard deviations. Correlations between two continuous variables were estimated as Pearson coefficients, and correlations between dichotomous and continuous variables as point-biserial coefficients. The differences in outcomes between mask types in possible interaction with mask-wearing duration were tested using analysis of co-variance (ANCOVA). Multivariate relationships between the predictor and the outcome measures were assessed within multiple linear regression models. For each predictor, we report its unstandardized coefficient B and its standard error, and for the model as a whole - the proportion of variance of the dependent variable explained by it $\left(R^{2}\right)$.

\section{Results}

\section{Descriptive statistics}

Of the 351 participants, $71.5 \%(N=251)$ were women, participants' age ranged from 18 to 84 years $(M=47.09$, $S D=17.87)$. The rate of chronic rhinitis and chronic sinusitis among the participants was $14.8 \%$ and $2.3 \%$, respectively. Seventy percent of participants answered "yes", $12 \%$ answered "no", and $18 \%$ answered "maybe" to the question, whether during the time of the pandemic they had 
experienced a change in their breathing. The median total SNOT-22 score was 13 , and $10 \%$ of the participants reported "significantly impaired QoL" (total SNOT-22>40) [10]. The distribution of the remaining research variables is presented in Table 1.

\section{Validity of the MaskSNOT questionnaire}

To establish the novel MaskSNOT scale's structural validity, we correlated its score with the scores of SNOT-22. The correlations were high: $r=0.85$ with the total SNOT-22 score, and $r=0.75,0.72,0.68,0.69,0.58$, and 0.46 with rhinologic symptoms, extranasal rhinologic symptoms, ear/facial symptoms, psychological dysfunction, and sleep dysfunction, respectively. Thus, the new scale's convergent validity was confirmed.

For the analysis of the mask-type effect, N95 types with and without a filter were combined because too few responders reported usage of each of these two types. No one reported wearing the "other" mask type. Analyses of co-variance of the MaskSNOT, total SNOT-22, and its subscales revealed that these outcomes were not affected by mask type or by the interaction between mask type and wearing duration. The majority change their masks once in a day or two. Mask usage was the highest in closed public places and outdoors and the lowest, as expected, at home.

\section{Univariate analyses}

As seen in Table 2, women had higher scores on the total SNOT-22 than men, on all of its subscales (especially those of psychological dysfunction and sleep dysfunction), and on the MaskSNOT. The presence of chronic rhinitis and sinusitis was related to higher levels of the total SNOT-22 score and two of its sub-scales (rhinologic and extranasal rhinologic symptoms). Rhinitis was also predictive of higher sleep dysfunction. Participants suffering from chronic pulmonary problems had higher
Table 1 Distribution of research variables

\begin{tabular}{|c|c|c|}
\hline Variable & Mean or percent & $\begin{array}{l}\text { Standard } \\
\text { deviation }\end{array}$ \\
\hline Gender $(1=$ female, $0=$ male $)$ & $71.5 \%$ & \\
\hline Age & 47.09 & 17.87 \\
\hline \multicolumn{3}{|l|}{ Chronic illnesses $(1=$ yes, $0=$ no $)$} \\
\hline Rhinitis & $14.8 \%$ & \\
\hline Sinusitis & $2.3 \%$ & \\
\hline Asthma or other lung diseases & $5.1 \%$ & \\
\hline Cardiac problem and/or elevated blood pressure & $10.8 \%$ & \\
\hline Other illness & $13.4 \%$ & \\
\hline No chronic health problem & $62.4 \%$ & \\
\hline $\begin{array}{l}\text { Continuous medical treatment due to the chronic airway } \\
\text { problem }\end{array}$ & $7.1 \%$ & \\
\hline Number of times a mask is changed during the day & 3.12 & 1.30 \\
\hline The average daily mask-use (hours) & 2.80 & 1.27 \\
\hline \multicolumn{3}{|l|}{ Frequency of mask use ${ }^{\#}$} \\
\hline At home & 1.19 & 0.53 \\
\hline During a social meeting & 3.65 & 1.23 \\
\hline During a family meeting & 2.31 & 1.33 \\
\hline At the workplace & 3.80 & 1.48 \\
\hline Outdoors & 4.48 & 0.81 \\
\hline In closed public places & 4.87 & 0.37 \\
\hline \multicolumn{3}{|l|}{ SNOT-22 } \\
\hline Total score & 18.10 & 16.91 \\
\hline Rhinologic symptoms & 3.74 & 4.89 \\
\hline Extranasal rhinologic symptoms & 1.61 & 2.68 \\
\hline Ear/facial symptoms & 2.79 & 3.89 \\
\hline Psychological dysfunction & 8.13 & 7.59 \\
\hline Sleep dysfunction & 5.67 & 6.10 \\
\hline MaskSNOT & 5.19 & 6.09 \\
\hline
\end{tabular}

${ }^{\#} 0$ —-not at all; 1—rarely; 2-sometimes; 3-often; 4-always 
Table 2 Correlations between the predictor and the outcome variables

\begin{tabular}{|c|c|c|c|c|c|c|c|}
\hline \multirow[t]{2}{*}{ Predictor variables } & \multicolumn{7}{|c|}{ Outcome measures } \\
\hline & SNOT-22 Total & $\begin{array}{l}\text { Rhinologic } \\
\text { symptoms }\end{array}$ & $\begin{array}{l}\text { Extranasal } \\
\text { rhinologic } \\
\text { symptoms }\end{array}$ & Ear/facial symptoms & $\begin{array}{l}\text { Psychologi- } \\
\text { cal dysfunc- } \\
\text { tion }\end{array}$ & Sleep dysfunction & MaskSNOT \\
\hline $\begin{array}{l}\text { Gender }(1=\text { female } \\
0=\text { male })\end{array}$ & $0.18 * * *$ & 0.06 & 0.03 & 0.07 & $0.23 * * *$ & $0.22 * * *$ & $0.15^{* *}$ \\
\hline Age & $-0.19 * * *$ & $-0.15 * *$ & $-0.15^{* *}$ & $-0.16^{* *}$ & $-0.20 * * *$ & $-0.13 *$ & $-0.14 * *$ \\
\hline \multicolumn{8}{|c|}{ Chronic illnesses $(1=$ yes, $0=$ no $)$} \\
\hline Rhinitis & $0.19 * * *$ & $0.28 * * *$ & $0.25^{* * *}$ & 0.10 & 0.09 & $0.17 * *$ & 0.05 \\
\hline Sinusitis & $0.11 *$ & $0.18 * * *$ & $0.16^{* *}$ & 0.06 & 0.03 & 0.09 & 0.07 \\
\hline $\begin{array}{l}\text { Asthma or other lung } \\
\text { diseases }\end{array}$ & 0.04 & 0.07 & 0.06 & 0.04 & 0.01 & 0.01 & $0.13 *$ \\
\hline $\begin{array}{l}\text { Cardiac problem and/ } \\
\text { or elevated blood } \\
\text { pressure }\end{array}$ & -0.06 & -0.02 & 0.00 & -0.07 & -0.07 & -0.04 & -0.01 \\
\hline Other illness & 0.00 & -0.05 & -0.02 & -0.04 & 0.05 & 0.07 & -0.02 \\
\hline $\begin{array}{l}\text { No chronic health } \\
\text { problem }\end{array}$ & -0.05 & $-0.11^{*}$ & -0.10 & 0.03 & -0.01 & -0.06 & -0.02 \\
\hline $\begin{array}{l}\text { Continuous medi- } \\
\text { cal treatment due to } \\
\text { the chronic airway } \\
\text { problem }\end{array}$ & 0.05 & $0.14 * *$ & $0.14 * *$ & 0.01 & 0.00 & 0.02 & 0.09 \\
\hline $\begin{array}{l}\text { Number of times a mask } \\
\text { is changed during the } \\
\text { day }\end{array}$ & $0.11 *$ & $0.12 *$ & $0.12 *$ & $0.14 *$ & 0.05 & 0.06 & -0.10 \\
\hline $\begin{array}{l}\text { The average daily mask- } \\
\text { use (hours) }\end{array}$ & 0.10 & $0.16 * *$ & $0.12 *$ & $0.14 * *$ & 0.07 & 0.00 & $0.15 * *$ \\
\hline \multicolumn{8}{|l|}{ Mask use $^{\#}$} \\
\hline At home & -0.01 & -0.04 & -0.07 & 0.01 & 0.04 & -0.02 & -0.02 \\
\hline $\begin{array}{l}\text { During a social meet- } \\
\text { ing }\end{array}$ & -0.03 & 0.03 & 0.02 & 0.00 & -0.07 & -0.04 & -0.09 \\
\hline $\begin{array}{l}\text { During a family meet- } \\
\text { ing }\end{array}$ & 0.00 & -0.04 & -0.02 & 0.00 & 0.00 & 0.01 & -0.07 \\
\hline At the workplace & 0.08 & 0.10 & 0.11 & 0.13 & 0.05 & -0.01 & 0.12 \\
\hline Outdoors & $-0.13 *$ & -0.10 & $-0.14 *$ & $-0.16^{* *}$ & -0.09 & -0.06 & $-0.14 * *$ \\
\hline In closed public places & $-0.13 *$ & -0.10 & $-0.16^{* *}$ & $-0.13^{*}$ & -0.10 & -0.05 & $-0.11^{*}$ \\
\hline \multicolumn{8}{|l|}{$* p<0.05$} \\
\hline \multicolumn{8}{|l|}{$* * p<0.01$} \\
\hline \multicolumn{8}{|l|}{$* * * p<0.001$} \\
\hline${ }^{\#} 0$ —not at all; 1 -rarely; & 2 -sometimes. 3 & ftem 1 & ways & & & & \\
\hline
\end{tabular}

MaskSNOT scores than others. Mask change frequency and daily use duration were positively correlated with all the total SNOT-22 score and three of its sub-scales: rhinologic, extranasal, and ear/facial symptoms. Also, usage duration was positively correlated with the MaskSNOT score. Mask-wearing in two contexts, outdoors and closed public places, correlated negatively with the total SNOT22 score, two of its sub-scales (extranasal rhinologic and ear/facial symptoms), and the MaskSNOT (Table 2).

\section{Multivariate analyses}

As seen in Table 2, each outcome was related to more than one predictor. To estimate the unique contribution of each predictor, multiple regression analysis was performed on each outcome. In order to ensure comparability of results, each outcome was regressed on all of the predictors that were found, in univariate analyses, to be significantly correlated with at least one outcome. The resulting models are presented in Table 3 . The only subject-related variables 


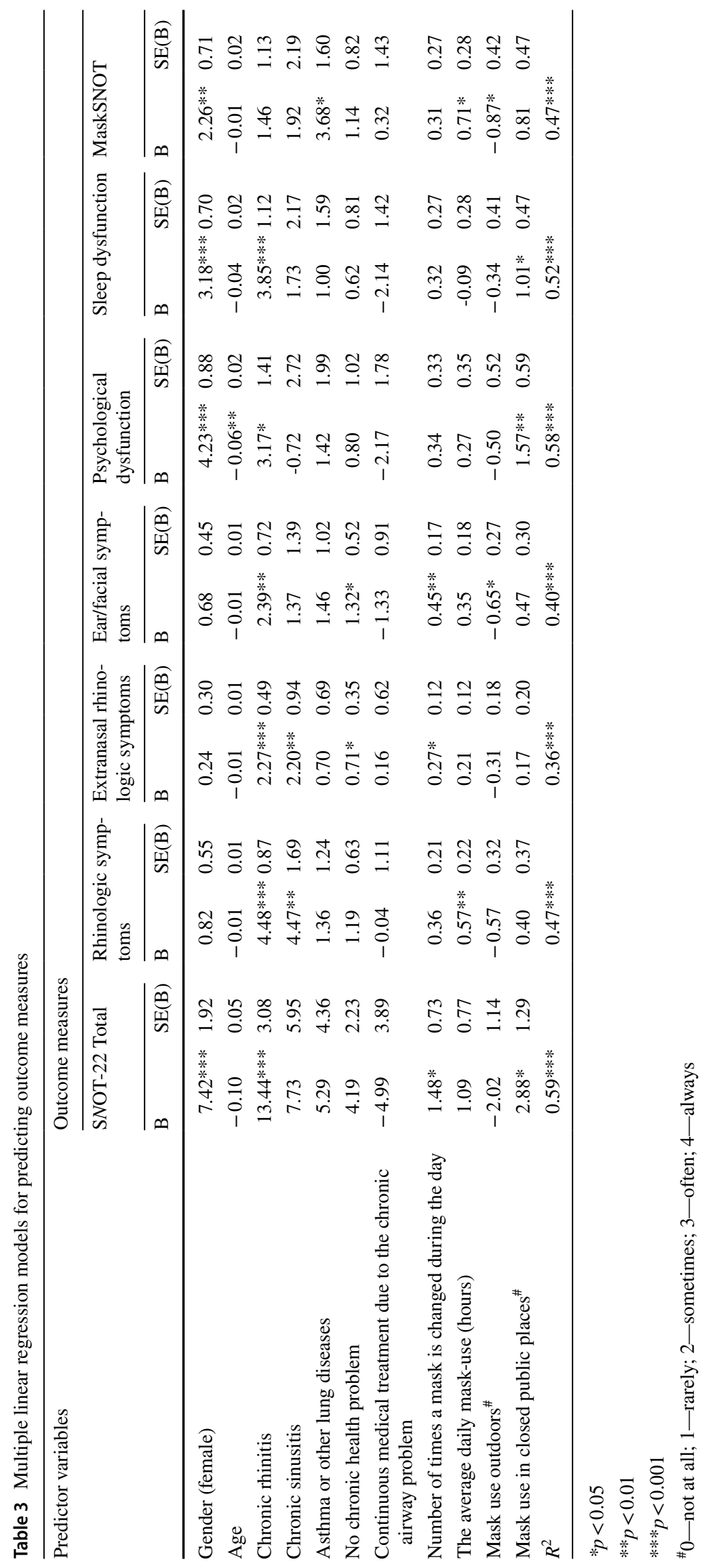


significantly associated with the reduced sinonasal QoL were female gender, younger age, a background of chronic rhinitis and sinusitis, and the mask-wearing average daily duration.

\section{Discussion}

The COVID-19 pandemic has had a global impact on human health. Aside from its immediate harm to those suffering from the disease, mandatory universal masking may have adverse effects on sino-nasal QoL and exacerbate chronic illnesses. The present study sought to assess this effect, including the differences between the mask types.

Our study cohort provides a good representation of the total population: the rates of chronic rhinitis $(14.8 \%)$ and chronic sinusitis $(2.3 \%)$ in our cohort correspond well with those reported in the literature [11,12]. Additionally, the percentage of our survey responders ever tested positive for COVID-19 (2.6\%) was similar to that in the total Israeli population during the time the survey was conducted [13].

The MaskSNOT scale constructed for evaluating the specific effect of facial masks on the sino-nasal QoLwas found structurally valid. It also correlated well with the total SNOT-22 score and each of its clustered domains. Therefore, the MaskSNOT can serve as a valuable adjunctive to the SNOT-22 in future studies of sinonasal QoL during the time of the COVID-19 pandemic.

The vast majority of the responders (70\%) reported experiencing a change in their breathing while wearing a face mask. This finding is in line with the relatively high median total SNOT-22 score (13) for the whole study cohort. In contrast, the normal SNOT-22 score in adults without sinonasal diseases is 7, according to Gillett et al. [14]. Thus, our results appear to highlight the negative impact of mask-wearing on the sinonasal QoL during the COVID-19 pandemic. Total SNOT-22, psychological and sleep dysfunction, as well as MaskSNOT scores, were higher among women compared to men. Our data are supported by previous studies dealing with the COVID-19 period and a large body of research reporting the increased prevalence of anxiety and sleep disorders among females [15-19]. Along with that, these gender differences can possibly reflect the more significant burden of familial responsibilities on females, particularly care for their children's emotional, physical, and intellectual well-being during a time when schools and kindergartens were closed.

As expected, participants suffering from chronic rhinitis and sinusitis demonstrated higher SNOT-22 scores, especially on the rhinologic and extranasal rhinologic symptoms sub-scores. Nevertheless, their QoL was not further affected by mask-wearing. This observation is in agreement with the data presented by Dror et al. [5] and can be attributed to the reduced exposure to allergens when using face masks. In contrast, responders with chronic pulmonary diseases were found to suffer more from the symptoms included in the MaskSNOT. This could be attributed to increased breathing effort and rebreathing of the exhaled air. The participants' age negatively correlated with all outcome measures. This is possibly a reflection of the increased social distancing among the elderly population, allowing them to minimize the use of face masks.

The average daily duration of mask-wearing strongly correlated with MaskSNOT scores, rhinologic, and ear/facial symptoms. The latter finding can be explained by the physical pressure that masks apply on the ears and the face. Another interesting finding is the negative correlation found between all outcome parameters (especially the total SNOT-22 score, extranasal rhinologic and ear/facial clusters, and the MaskSNOT) and mask-wearing outdoors and in closed public places. Participants used masks in these circumstances more than in other ones. Air conditions in these places cannot be adjusted individually, and face masks may help create a personal, more humid and hot micro-environment.

In our study, the mask type was not found to significantly affect the checked QoL parameters. Furthermore, previous research on the antiviral efficacy of different mask types and materials has yielded contradicting and equivocal findings [20-22]. From this standpoint, it is unclear whether there are advantages to choosing one type of mask over another. Interestingly, the responders wore their masks less frequently during social meetings than outdoors or in closed public places. It can be potentially attributable either to maskrelated communication disturbance or limited enforcement in the social meeting environment.

The multivariate analyses revealed that women exhibited reduced sinonasal QoL, especially in terms of psychological and sleep dysfunction, compared to men. This can be argued to reflect the above-mentioned female predisposition to depression, anxiety and sleep disturbances [17-19]. In contrast, this could be caused by the responders' difficulty in differentiating between their baseline condition and recent changes related to the mask-use in the COVID-19 pandemic period. The latter statement could also explain the strong correlation between sinonasal QoL and background of chronic rhinitis and sinusitis and the association between the psychologic disorder outcome variable and younger age $[18,23]$. The mask-wearing average daily duration was associated mostly with the rhinologic symptoms and those included in the MaskSNOT score.

\section{Conclusion}

The majority of our survey's responders reported reduced sinonasal QoL in the COVID-19 pandemic period. Based on the results obtained using the MaskSNOT, this change 
in the QoL is attributable to mask-wearing, especially for a prolonged time, irrespective of the existing mask types. We believe that these findings will encourage medical companies to produce more "airway-minded" personal protection equipment.

Supplementary Information The online version contains supplementary material available at https://doi.org/10.1007/s00405-021-06752-2.

Author contributions AP-F: study design, data collection, and writing. OA: study design, interpretation of data, and editing. IR: data collection, analysis, and writing. RM-S: study design, data collection, and editing. EEA: data collection, analysis, and editing. AY: study design, data collection, and drafting the article.

Funding No funds, grants, or other support was received.

Availability of data and material DOI. 10.7303/syn24611029.

\section{Declarations}

Conflict of interest The authors have no relevant financial or non-financial interests to disclose.

Ethics approval This study was performed in line with the principles of the Declaration of Helsinki. Approval was granted by the Tel-Aviv University Ethics Committee (\#1-0002166).

Consent to participate Informed consent was obtained from all individual participants included in the study.

\section{References}

1. Plana D, Tian E, Cramer AK et al (2020) Assessing the quality of nontraditional N95 filtering face-piece respirators available during the COVID-19 pandemic. medRxiv. https://doi.org/10.1101/2020. 07.25.20161968

2. Sublett JW, Bernstein DI (2011) Occupational rhinitis. Immunol Allergy Clin North Am 31(787-96):vii. https://doi.org/10.1016/j. iac.2011.07.007

3. de Dantas IP, Valera FCP, Zappelini CEM, Anselmo-Lima WT (2013) Prevalence of rhinitis symptoms among textile industry workers exposed to cotton dust. Int Arch Otorhinolaryngol 17:2630. https://doi.org/10.7162/S1809-97772013000100005

4. Veloso-Teles R, Cerejeira R, Roque-Farinha R, von Buchwald C (2018) Higher prevalence of nasal polyposis among textile workers: an endoscopic based and controlled study. Rhinology 56:99-105. https://doi.org/10.4193/Rhin17.228

5. Dror AA, Eisenbach N, Marshak T et al (2020) Reduction of allergic rhinitis symptoms with face mask usage during the COVID-19 pandemic. J Allergy Clin Immunol Pract 8:3590-3593. https://doi. org/10.1016/j.jaip.2020.08.035

6. Shapira Galitz Y, Halperin D, Bavnik Y, Warman M (2016) Sinonasal outcome test-22: translation, cross-cultural adaptation, and validation in hebrew-speaking patients. Otolaryngol Head Neck Surg 154:951-956. https://doi.org/10.1177/0194599816629378

7. Morley AD, Sharp HR (2006) A review of sinonasal outcome scoring systems-which is best? Clin Otolaryngol 31:103-109. https://doi.org/10.1111/j.1749-4486.2006.01155.x

8. Rudmik L, Hopkins C, Peters A et al (2015) Patient-reported outcome measures for adult chronic rhinosinusitis: a systematic review and quality assessment. J Allergy Clin Immunol 136:15321540.e2. https://doi.org/10.1016/j.jaci.2015.10.012

9. DeConde AS, Mace JC, Bodner T et al (2014) SNOT-22 quality of life domains differentially predict treatment modality selection in chronic rhinosinusitis. Int Forum Allergy Rhinol 4:972-979. https://doi.org/10.1002/alr.21408

10. Fokkens WJ, Lund VJ, Hopkins C et al (2020) European position paper on rhinosinusitis and nasal polyps 2020. Rhinology 58:1-464. https://doi.org/10.4193/Rhin20.600

11. Seidman MD, Gurgel RK, Lin SY et al (2015) Clinical practice guideline: allergic rhinitis. Otolaryngol Head Neck Surg 152:S143. https://doi.org/10.1177/0194599814561600

12. Dietz de Loos D, Lourijsen ES, Wildeman MAM et al (2019) Prevalence of chronic rhinosinusitis in the general population based on sinus radiology and symptomatology. J Allergy Clin Immunol 143:1207-1214. https://doi.org/10.1016/j.jaci.2018.12. 986

13. Corona virus in Israel - general situation Web site. Available at: https://datadashboard.health.gov.il/COVID-19/general. Accessed 26 October 2020.

14. Gillett S, Hopkins C, Slack R, Browne JP (2009) A pilot study of the SNOT 22 score in adults with no sinonasal disease. Clin Otolaryngol 34:467-469. https://doi.org/10.1111/j.1749-4486.2009. 01975.x

15. Bigalke JA, Greenlund IM, Carter JR (2020) Sex differences in self-report anxiety and sleep quality during COVID-19 stayat-home orders. Biol Sex Differ 11:56. https://doi.org/10.1186/ s13293-020-00333-4

16. Huang Y, Zhao N (2020) Generalized anxiety disorder, depressive symptoms and sleep quality during COVID-19 outbreak in China: a web-based cross-sectional survey. Psychiatry Res 288:112954. https://doi.org/10.1016/j.psychres.2020.112954

17. Grandner MA, Martin JL, Patel NP et al (2012) Age and sleep disturbances among American men and women: data from the U.S. Behav Risk Factor Surveill Syst Sleep 35:395-406. https:// doi.org/10.5665/sleep. 1704

18. Salk RH, Hyde JS, Abramson LY (2017) Gender differences in depression in representative national samples: meta-analyses of diagnoses and symptoms. Psychol Bull 143:783-822. https://doi. org/10.1037/bul0000102

19. Bandelow B, Michaelis S (2015) Epidemiology of anxiety disorders in the 21st century. Dialogues Clin Neurosci 17:327-335

20. MacIntyre CR, Seale H, Dung TC et al (2015) A cluster randomised trial of cloth masks compared with medical masks in healthcare workers. BMJ Open 5:e006577. https://doi.org/10. 1136/bmjopen-2014-006577

21. Bartoszko JJ, Farooqi MAM, Alhazzani W, Loeb M (2020) Medical masks vs N95 respirators for preventing COVID-19 in healthcare workers: a systematic review and meta-analysis of randomized trials. Influenza Other Respi Viruses 14:365-373. https:// doi.org/10.1111/irv.12745

22. Wilson AM, Abney SE, King M-F et al (2020) COVID-19 and non-traditional mask use: how do various materials compare in reducing the infection risk for mask wearers? J Hosp Infect. https://doi.org/10.1016/j.jhin.2020.05.036

23. Olaya B, Moneta MV, Miret M et al (2018) Epidemiology of panic attacks, panic disorder and the moderating role of age: results from a population-based study. J Affect Disord 241:627-633. https://doi.org/10.1016/j.jad.2018.08.069

Publisher's Note Springer Nature remains neutral with regard to jurisdictional claims in published maps and institutional affiliations. 